\title{
Influence of Pre-operative Septal Mitral Annular Plane Systolic Excursion on the Outcome of Transfemoral Transcatheter Aortic Valve Implantation
}

Mohammad El-Garhy ${ }^{1,2^{*}}$, Tamer Owais ${ }^{1,3}$, Andreas Wagner ${ }^{1}$, Mathias Schreiber ${ }^{1}$, Thomas Kuntze ${ }^{1}$ and Jürgen Fuchs ${ }^{4}$

${ }^{1}$ Department of Cardiology, Heart Centre, Central Clinic in Bad Berka, Zentralklinik Bad Berka, Germany

${ }^{2}$ Department of Cardiology, Minia University, Egypt

${ }^{3}$ Department of Cardiothoracic Surgery, Cairo University, Egypt

${ }^{4}$ Department of Anesthesia, Zentralklinik Bad Berka, Germany

*Corresponding author: Mohammad El-Garhy, Department of Cardiology, Heart Centre, Central Clinic in Bad Berka, Robert-Koch Allee 9 , 99437 Zentralklinik Bad Berka, Germany, Tel: 493418650; E-mail: drmng81@yahoo.com

Received: July 03, 2018; Accepted: July 23, 2018; Published: July 26, 2018

Copyright: (c) $2018 \mathrm{El-Garhy} \mathrm{M,} \mathrm{et} \mathrm{al.} \mathrm{This} \mathrm{is} \mathrm{an} \mathrm{open-access} \mathrm{article} \mathrm{distributed} \mathrm{under} \mathrm{the} \mathrm{terms} \mathrm{of} \mathrm{the} \mathrm{creative} \mathrm{commons} \mathrm{attribution} \mathrm{license,} \mathrm{which} \mathrm{permits} \mathrm{unrestricted}$ use, distribution, and reproduction in any medium, provided the original author and source are credited.

\begin{abstract}
Objectives: The assessment of left ventricle longitudinal systolic function should be done routinely by echocardiographic assessment of aortic stenosis. In this study, we examined the predictive value of septal mitral annular plane systolic excursion (SMAPSE) in patients undergoing transfemoral transcatheter aortic valve Implantation (TF-TAVI).
\end{abstract}

Methods: Out of 653 patients operated as TF TAVI in the period between January 2016 and December 2017 in central hospital, Bad Berka, Germany, we included 315 patients who respected our inclusionlexclusion criteria. Patients were stratified into two groups according to their baseline MAPSE. Group I included patients with MAPSE $>7 \mathrm{~mm}$ and group II included patients with MAPSE $\leq 7 \mathrm{~mm}$. We compared between both groups as regards to improvement of symptoms, 3 months- mortalities and hospital readmissions because of heart failure. Seventeen patients were absent from the 3 months follow up scheduled appointment and consequently were excluded from our statistics.

Results and Discussion: During the 3 months follow-up period two mortalities were recorded and it concerned two patients in group II ( $0 \%$ group I vs $1,8 \%$ group II, p value 0.1$)$. Five patients $(1.5 \%)$ were readmitted because of heart failure, 1 patient in group I $(0.4 \%)$ and 4 patients group II (3.6\%), p value 0.052 . The incidence of combined hospital readmissions and mortalities was obviously lower in patients with MAPSE $>7 \mathrm{~mm}(0.4 \%$ in group I vs $5.4 \%$, $p$ value 0.008 ). There was no reported statistically significant difference in the improvement of symptoms between the two groups. Improvement in symptoms was noticed in a total of 240 patients $(76 \%)$ in the whole study group.

Conclusion: Reduced SMAPSE associated with more mortalities and hospital readmissions after TF-TAVI but cannot predict the improvement of symptoms postoperative.

Keywords: Transcatheter Aortic Valve Implantation (TAVI); Diastolic heart failure; Mitral Annular Plane Systolic Excursion (MAPSE); Left Ventricular Longitudinal Systolic Function (LVLSF)

\section{Introduction}

Transcatheter Aortic Valve Implantation (TAVI) is rising to be a standard of care for severe symptomatic aortic valve stenosis in highand intermediate- surgical risk patients [1,2]. Long standing aortic stenosis expresses a non-physiologic LV hypertrophy, which results in subendocardial ischemia and later on develops interstitial myocardial fibrosis [3]. Both of them pass through two phases: an early reversible phase where the longitudinal and spiral fibers are affected and consequently reduce the longitudinal systolic function [4]. In the later phase; severe myocardial fibrosis induced by severe aortic stenosis was shown to be irreversible even after aortic valve replacement [5]. Other studies reported a relevant implication of the degree of myocardial fibrosis on the clinical status and long-term survival after aortic valve replacement [6]. Mitral Annular Plane Systolic Excursion (MAPSE) is an easily acquired metric of left ventricular longitudinal systolic function (LVLSF). Moreover, previous studies showed that reduced MAPSE was an indicator of worse outcome after surgical aortic valve replacement and MAPSE more than $7 \mathrm{~mm}$ was associated with satisfactory functional improvement after aortic valve replacement [7-10]. We aimed in this study to investigate the impact of reduced MAPSE on improvement of symptoms, 3 months mortality and hospital readmission because of heart failure in patients undergoing TAVI.

\section{Research Methodology}

We conducted a retrospective study for patients operated with TAVI between January 2016 and December 2017 in the central hospital in Bad Berka, Germany. Patients with previous myocardial infarction, prior heart surgery or other severe valvular abnormalities were excluded. Furthermore, we excluded from our statistics two intraoperative mortalities and seventeen patients who did not show-up for the 3 months scheduled follow-up appointment. The remaining 315 patients were divided into two groups: group I with MAPSE $>7 \mathrm{~mm}$ and group II with MAPSE $\leq 7 \mathrm{~mm}$. Our clinical endpoints were 
Citation: El-Garhy M, Owais T, Wagner A, Schreiber M, Kuntze T, et al. (2018) Influence of Pre-operative Septal Mitral Annular Plane Systolic Excursion on the Outcome of Transfemoral Transcatheter Aortic Valve Implantation. J Cardiovasc Dis Diagn 6: 330. doi: $10.4172 / 2329-9517.1000330$

Page 2 of 4

improvement of the symptoms, 3-months mortalities and hospital readmissions due to heart failure. All decisions regarding the method of aortic valve replacement (i.e., conventional vs. TAVI), choice of the TAVI prosthesis (i.e., balloon-expandable vs. self-expandable) were decided by the heart team including cardiologists, cardiac surgeons and anesthesiologists. All patients were operated using general anesthesia. Edwards SAPIEN, SAPIEN XT and SAPIEN 3 THVs (Irvine, CA, USA), Medtronic CoreValve THV (Minneapolis, MN, USA), Symetis ACURATE TA and neo THV's (Ecublens, $\mathrm{CH}$ ) were implanted by transfemoral approach. The echocardiographic data were obtained from the preoperative and 3 months follow up echocardiography to document the LV ejection fraction, MAPSE, LV end-diastolic diameter, left atrial size, interventricular septal thickness (end diastolic), Posterior wall thickness (end diastolic). The clinical follow-ups were performed through office visits including transthoracic echocardiography.

\section{Statistical analysis}

Continuous variables are expressed as means and standard deviations. Parametric and non-parametric tests were used based on sample size and normality of distribution. The categorical data are expressed as percentages. Fisher exact and chi square were used to identify differences between both groups. All analysis was done using SPSS statistical software (IBM Corp. Released 2013. IBM SPSS Statistics for Windows, Version 22.0. Armonk, NY: IBM Corp).

\section{Results}

Our retrospective results showed the following: out of 315 patients, 205 patients had MAPSE $>7 \mathrm{~mm}$ ('Group I' 65\%) and 110 had MAPSE $\leq 7 \mathrm{~mm}$ ('Group II' 35\%). Table 1 provides the baseline clinical data according to MAPSE. Patients with MAPSE $\leq 7 \mathrm{~mm}$ were more symptomatic (NYHA III-IV 66.6\% in Group I and $90.9 \%$ in Group II, p-value 0.004 ), otherwise there was no statistical significant difference between both groups as regards to the baseline characteristics. The echocardiographic findings and the clinical outcomes are shown in Table 2. An overview of our summative results of both groups was as follows; 3 -months mortality was $0.6 \%$, the hospital readmission was $1.3 \%$ and improvements of the heart failure symptoms were recorded in $76 \%$. There were no statistically significant differences between the groups regarding improvement of the symptoms. This could be shown in the following results: 158 (77\%) patients in Group I and 82 (74.5\%) patients in Group II observed a remarkable improvement of the NYHA functional class (at least one NYHA class) after TAVI, p-value $=0.09$ (Table 2).

Our statistically significant domain of results included 3- months hospital readmission due to heart failure as well as the 3- months mortality. In Group II (MAPSE $\leq 7 \mathrm{~mm}$ ) showed a higher rate of hospital readmissions due to heart failure $(0.4 \%$ of group I vs $3.6 \%$ of group II, $\mathrm{p}$ value 0.52 ). Two mortalities were recorded in the study patients and both were in group II ( $0 \%$ of group I and $1.8 \%$ of group II, $\mathrm{p}$ value 0.1$)$. The incidence of combined hospital readmissions and mortalities was obviously lower in patients with MAPSE $>7 \mathrm{~mm}(0.4 \%$ in group I vs $5.4 \%$, p value 0.008 ) (Figure 1 ).

\section{Discussion}

We focused in this study on the 3 months MAPSE related results concerning improvement of symptoms, readmission and mortality.

\begin{tabular}{|c|c|c|c|c|}
\hline Clinical Data & Total & $\begin{array}{l}\text { Group I } \\
\mathrm{n}=205\end{array}$ & $\begin{array}{l}\text { Group II } \\
n=110\end{array}$ & P - Value \\
\hline Age (years \pm SD) & $79.60 \pm 4.2$ & $79.50 \pm 5.3$ & $79.84 \pm 5.9$ & 0.9 \\
\hline Male n (\%) & $\begin{array}{l}178 \\
(56.5 \%)\end{array}$ & $120(58 \%)$ & $58(52 \%)$ & 0.4 \\
\hline COPD n (\%) & $72(22.8 \%)$ & $45(22 \%)$ & $27(25 \%)$ & 0.5 \\
\hline CRF $>$ II n (\%) & $\begin{array}{l}111 \\
(35.2 \%)\end{array}$ & $70(34 \%)$ & $41(37 \%)$ & 1.0 \\
\hline NYHA III- IV n (\%) & $\begin{array}{l}211 \\
(66.9 \%)\end{array}$ & $\begin{array}{l}111 \\
(66.6 \%)\end{array}$ & $\begin{array}{l}100 \\
(90.9 \%)\end{array}$ & 0.004 \\
\hline $\begin{array}{l}\text { Previous myocardial } \\
\text { infarction } \mathrm{n}(\%)\end{array}$ & $90(28.5 \%)$ & $60(29 \%)$ & $30(27 \%)$ & 0.6 \\
\hline $\mathrm{PH} n(\%)$ & $186(59 \%)$ & $113(55 \%)$ & $73(59 \%)$ & 0.8 \\
\hline $\mathrm{BMI}>30 \mathrm{n}(\%)$ & $71(22.5 \%)$ & $43(21 \%)$ & $28(25 \%)$ & 0.9 \\
\hline $\begin{array}{ll}\mathrm{Hb} & {[\mathrm{mmol} /]} \\
\text { (preoperative) } & \end{array}$ & $8.36 \pm 1.4$ & $8.4 \pm 2.0$ & $8.3 \pm 1.0$ & 1.0 \\
\hline $\begin{array}{l}\text { Log EuroScore (mean } \pm \\
\text { SD) }\end{array}$ & $41 \pm 7$ & $40 \pm 8$ & $43 \pm 7$ & 0.2 \\
\hline
\end{tabular}

N number; NYHA New York heart association; PH pulmonary hypertension; BMI body mass index; $\mathrm{Hb}$ haemoglobin; SD standard deviation.

Table 1: Comparison between the groups as regard baseline characteristics.

\begin{tabular}{|l|l|l|l|l|}
\hline Variables & $\begin{array}{l}\text { Total } \\
\mathbf{N}=\mathbf{3 1 5}\end{array}$ & $\begin{array}{l}\text { Group I } \\
\mathbf{N}=\mathbf{2 0 5}(\mathbf{6 5} \%)\end{array}$ & $\begin{array}{l}\text { Group II } \\
\mathbf{N}=\mathbf{1 1 0}(\mathbf{3 5} \%)\end{array}$ & $\mathbf{p}$ - Value \\
\hline EF & $55.9 \pm 7$ & $56.5 \pm 5.2$ & $54.2 \pm 3.5$ & 0.08 \\
\hline $\begin{array}{l}\text { MG AV mmHg } \\
\text { SD }\end{array}$ & $49.6 \pm 16$ & $50.1 \pm 14.6$ & $49 \pm 20$ & 0.4 \\
\hline LA mm & $41.1 \pm 4.5$ & $40.8 \pm 5.1$ & $41.8 \pm 3.1$ & 0.07 \\
\hline LVEDD mm & $46.6 \pm 6.7$ & $45.5 \pm 6.1$ & $49 \pm 6.4$ & 0.06 \\
\hline SWT & $13.8 \pm 2.6$ & $13.7 \pm 2.4$ & $14.5 \pm 2$ & 0.2 \\
\hline PWT & $13 \pm 2.5$ & $13.2 \pm 2.5$ & $13 \pm 1.8$ & 0.1 \\
\hline MAPSE & $9 \pm 3$ & $10.5 \pm 2.6$ & $6.1 \pm 0.8$ & 0.002 \\
\hline $\begin{array}{l}\text { Improvement of } \\
\text { MAPSE }\end{array}$ & $196(62 \%)$ & $133(64.8 \%)$ & $63(57.2 \%)$ & 0.08 \\
\hline $\begin{array}{l}\text { Improvement of } \\
\text { NYHA }\end{array}$ & $240(76 \%)$ & $158(77 \%)$ & $82(74.5 \%)$ & 0.09 \\
\hline $\begin{array}{l}\text { Readmission to } \\
\text { hospital }\end{array}$ & $5(1.5 \%)$ & $1(0.4 \%)$ & $4(3.6 \%)$ & 0.03 \\
\hline $\begin{array}{l}\text { 3-months } \\
\text { Mortality }\end{array}$ & 2 (0.6\%) & 0 & $2(1.8 \%)$ & 0.05 \\
\hline $\begin{array}{l}\text { N number; EF ejection fraction; } \\
\text { MAPSE mitral annular plane systolic excursion; PWT posterior wall thickness. }\end{array}$ \\
\hline
\end{tabular}

Table 2: Difference between the two groups as regard echocardiographic parameters and clinical end points. 
Citation: El-Garhy M, Owais T, Wagner A, Schreiber M, Kuntze T, et al. (2018) Influence of Pre-operative Septal Mitral Annular Plane Systolic Excursion on the Outcome of Transfemoral Transcatheter Aortic Valve Implantation. J Cardiovasc Dis Diagn 6: 330. doi: $10.4172 / 2329-9517.1000330$

Page 3 of 4

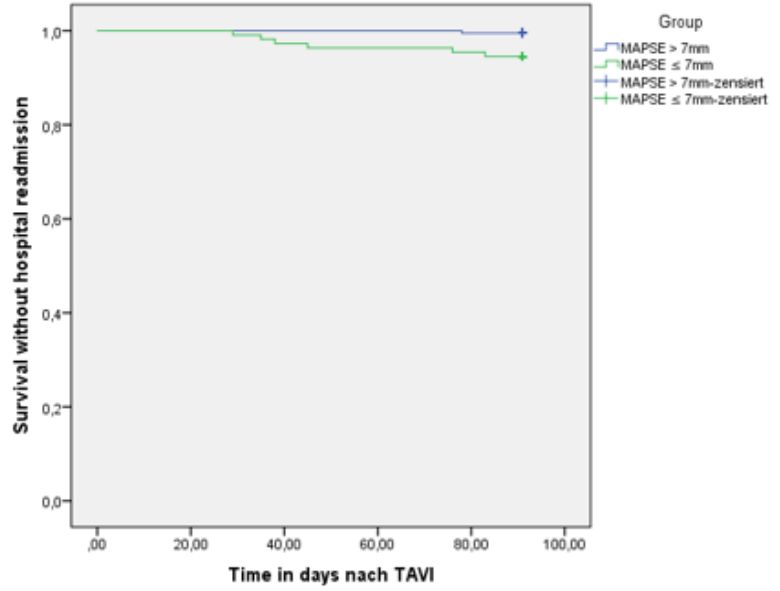

Figure 1: Kaplan-Meier survival curve shows significant difference between both groups as regard 3 months survival without hospital readmissions ( $\mathrm{p}$ value 0.004 )

Our point of view in limiting our follow to 3 months was related to our logistic institutional schedule for follow up after TAVI procedures to 3 months. However, we are aiming to extend our follow up to one year after publishing these interesting and encouraging results.

Our Choice of $7 \mathrm{~mm}$ MAPSE as a cut off point for the prediction of these results was based upon the presence of a previously published article in 2009 and 2013 by Weidemann and Hu K respectively who also took the $7 \mathrm{~mm}$ as a cut-off point. Unexpectedly, we obtained noncoinciding results with both of them concerning improvement of symptoms $[5,11]$.

Concerning our results in the domain of improvement of heart failure symptoms after TAVI, we recorded no significant difference between the two groups of our study. Unlikely, Weidemann who included fifty-eight patients in his study showed that MAPSE $>7 \mathrm{~mm}$ had a very good diagnostic utility to predict the improved NYHA class. However, it is worth mentioning that we noted a remarkable difference in our study's design and methodology when compared to his study. For example, Weidemann is a single center prospective study, his study group was operated upon surgically and he stratified his study population as well in to groups according to the degree of fibrosis not according to MAPSE as in our study. Another explanation for the rapid improvement of the symptoms in the patients with reduced MAPSE in our study population was that surgical aortic valve replacement is associated with more heart burden and need consequently slower improvement of the functional capacity. Furthermore, the decrease of MAPSE maybe related not only to irreversible myocardial fibrosis but also to the degree of aortic valve obstruction $[4,5]$.

MAPSE is a simple and highly accessible validated parameter of LVLSF, which could be obtained in almost all patients, even if the endocardial border is not clear $[10,11]$. We constricted the evaluation of LVLSF in our study to sMAPSE, as it is measured routinely in our institution for all the patients with aortic stenosis. Our hypothesis that sMAPSE is sufficient to assess LVLSF, was based on the work of Luszczak et al. which demonstrated that MAPSE is not inferior to global longitudinal peak strain to assess LVLSF [10,11]. Another supporter of our results is Takeda et al. who concluded that sMAPSE could not predict the deterioration of symptoms in the asymptomatic aortic stenosis patients [4]. However, in his study, lateral MAPSE was an independent predictor of the development of symptoms [4]. Septal MAPSE could be affected by many factors such as pulmonary hypertension with paradoxical septal motion, post-operative and after myocardial infarction. Therefor global MAPSE should be the standard for assessment of the LVLSF rather than the sMAPSE. The improvement of the NYHA class (at least 1 NYHA class) was recorded in $76 \%$ of our patients. The improvement of the functional class in previous studies ranging between $60 \%$ to $86 \%$ was due to many factors such as risk score, frailty, comorbidities, aortic stiffness, myocardial fibrosis and low gradient severe aortic stenosis. We included in this study a higher portion of higher risk patients (Log EuroScore 30.8\%), $23.7 \%$ of patients had COPD and $45 \%$ had atrial fibrillation, these factors were independently associated with residual impairment of functional capacity after TAVI [12-15].

Our results in the domain of worsening of the clinical outcome in patients with reduced MAPSE coincide with Matos and El-Battrawy. However, we focused our study only on patients with predominant aortic stenosis but El-Battrawy focused on predominant Takotsubo syndrome. This could be pathophysiologically explained by the fact that reduced LVLSF is an early marker of myocardial dysfunction before the deterioration of radial and global systolic function.

The Improvement of the symptoms after a complex intervention such as TAVI is considered to be a more persuasive reason for high risk patients rather than the prolongation on their lifespan. Should MAPSE be the only parameter? Puri et al. replied to our question by publishing in 2013 his article, that combining TAVI scores along with frailty parameters, echocardiographic parameters and the presence of specific organ failure may be more accurate to predict TAVI-related futility $[14,15]$.

\section{Conclusion and Study Limitations}

Reduced MAPSE after TF-TAVI cannot predict the improvement of the symptoms and could be associated with higher hospital readmissions due to heart failure.

The limitations of our study are being a retrospective observational study, which lead to lack of other echocardiographic confounders as tissue Doppler parameters of longitudinal systolic function and lateral MAPSE. Otherwise some clinical confounding factors such as medication history were not studied and therefore a multivariate analysis could not be done. Finally, we encountered unfortunately a $5 \%$ lost follow up.

\section{Funding}

None.

\section{References}

1. Leon MB, Smith CR, Mack MJ, Makkar RR, Svensson LG, et al. (2016) Transcatheter or surgical aortic-valve replacement in intermediate-risk patients. N Engl J Med 374: 1609-1620.

2. Goleski PJ, Reisman M, Don CW (2016) Reversible thrombotic aortic valve restenosis after valve-in-valve transcatheter aortic valve replacement. JACC Cardiovasc Interv 91: 165-168.

3. Golia G, Milano AD, Dodonov M, Bergamini C, Faggian G (2011) Influence of myocardial fibrosis on left ventricular hypertrophy in patients with symptomatic severe aortic stenosis. Cardiology 120: 139145 . 
Citation: El-Garhy M, Owais T, Wagner A, Schreiber M, Kuntze T, et al. (2018) Influence of Pre-operative Septal Mitral Annular Plane Systolic Excursion on the Outcome of Transfemoral Transcatheter Aortic Valve Implantation. J Cardiovasc Dis Diagn 6: 330. doi: $10.4172 / 2329-9517.1000330$

Page 4 of 4

4. Takeda S, Rimington H, Smeeton N, Chambers J (2001) Long axis excursion in aortic stenosis. Heart 86: 52-56.

5. Weidemann F, Herrmann S, Störk S, Niemann M, Frantz S, et al. (2009) Impact of myocardial fibrosis in patients with symptomatic severe aortic stenosis. Circulation 18: 120: 577-584.

6. Milano AD, Faggian G, Dodonov M, Golia G, Tomezzoli A, et al. (2012) Prognostic value of myocardial fibrosis in patients with severe aortic valve stenosis. J Thorac Cardiovasc Surg 144: 830-837.

7. Matos JD, Kiss JE, Locke AH, Goldberg RI, Mukamal K, et al. (2017) Relation of the mitral annular plane systolic excursion to risk for intervention in initially asymptomatic patients with aortic stenosis and preserved systolic function. Am J Cardiol 120: 2031-2034.

8. El-Battrawy I, Ansari U, Lang S, Fastner C, Zhou X, et al. (2018) Risk stratification in Takotsubo Syndrome: A role of mitral annular plane systolic excursion. QJM 111: 231-236.

9. Chin CWL, Everett RJ, Kwiecinski J, Vesey AT, Yeung E, et al. (2017) Myocardial fibrosis and cardiac decompensation in aortic stenosis. JACC Cardiovasc Imaging 10: 1320-1333.

10. Luszczak J, Olszowska M, Drapisz S, Plazak W, Kaznica-Wiatr M, et al. (2013) Assessment of left ventricle function in aortic stenosis: mitral annular plane systolic excursion is not inferior to speckle tracking echocardiography derived global longitudinal peak strain. Cardiovasc Ultrasound 11: 45.
11. Hu K, Liu D, Herrmann S, Niemann M, Gaudron PD, et al. (2013) Clinical implication of mitral annular plane systolic excursion for patients with cardiovascular disease. European Heart Journal Cardiovascular Imaging 14: 205-212.

12. Poulsen SH, Søgaard P, Nielsen-Kudsk JE, Egeblad H (2007) Recovery of left ventricular systolic longitudinal strain after valve replacement in aortic stenosis and relation to natriuretic peptides. J Am Soc Echocardiogr 20: 877-884.

13. Abdelghani M, Cavalcante R, Miyazaki Y, De Winter RJ, Sarmento-Leite $\mathrm{R}$, et al. (2017) Prevalence, predictors, and prognostic implications of residual impairment of functional capacity after transcatheter aortic valve implantation. Clin Res Cardiol 106: 752-759.

14. Puri R, Iung B, Cohen DJ, Rodes-Cabau J (2016) TAVI or No TAVI: identifying patients unlikely to benefit from transcatheter aortic valve implantation. Eur Heart J 37: 2217-2225.

15. Reynolds MR, Magnuson EA, Wang K, Thourani VH, Williams M, et al. (2012) Health-related quality of life after transcatheter or surgical aortic valve replacement in high-risk patients with severe aortic stenosis: Results from the PARTNER (Placement of aortic transcatheter valve) Trial (Cohort A). J Am Coll Cardiol 60: 548-558. 